\title{
Clitoria ternatea anthocyanin extract suppresses inflammation in carrageenan- induced rat paw edema via down-regulating genes of phosphoinositide 3-kinase signaling pathway
}

\author{
${ }^{1,2 *}$ Yanti, ${ }^{1}$ Sabella, D., ${ }^{1}$ Gunawan, A.W. and ${ }^{1,2}$ Lay, B.W. \\ ${ }^{1}$ Faculty of Biotechnology, Atma Jaya Catholic University of Indonesia, Jakarta 12930, Indonesia \\ ${ }^{2}$ Research Center for Indonesian Spices, Atma Jaya Catholic University of Indonesia, Jakarta 12930, \\ Indonesia
}

\section{Article history:}

Received: 16 January 2020

Received in revised form: 11 March 2020

Accepted: 14 March 2020

Available Online: 8 March 2020

\section{Keywords:}

Clitoria ternatea

anthocyanin,

Gene expression,

PI3K signaling pathway,

Inflammation,

Edema rats

DOI:

https://doi.org/10.26656/fr.2017.4(4).028

\begin{abstract}
Inflammation is characterized by an increase in vascular permeability leading to edema formation. Phosphoinositide 3-kinase (PI3K) signaling pathway plays the main role to regulate several key events in the inflammatory response to damage and infection. Our previous in silico study showed that anthocyanin and ternatin flavonoids may offer antiinflammatory potential via molecular docking model. Here, we explored whether Clitoria ternatea anthocyanin extract (CTA) had protective activity against inflammation via regulating gene expression related to PI3Ks in rat edema. CTA was extracted in methanol and acetone using maceration and lyophilization. Edema model was done by injecting carrageenan into rat paw. Oral supplementation of CTA $(100,250$, and $500 \mathrm{mg} / \mathrm{kg} \mathrm{BW})$ and ibuprofen standard ( $15 \mathrm{mg} / \mathrm{kg} \mathrm{BW})$ was given to rats daily for 7 days. After sample treatment, the right hind paw of the rat was injected with carrageenan to induce edema condition. The ability of CTA to suppress the inflammation in paw edema was measured by quantifying the increased percentage of rat paw volume at 1,3 , and $5 \mathrm{hrs}$ after injection. Rat paw tissue was collected, and gene expression related to PI3Ks, i.e. Akt1, $P K B$, Ilk, Pdk2, Pik3Ca, and p53 was determined using quantitative PCR (qPCR). Carrageenan showed a time-dependent increase in volume of rat paw starting from injection until the next $5 \mathrm{hrs}$. It reached maximum volume increase of rat paw at $3 \mathrm{hrs}$ after injection. CTA treatment had a dose-dependent pattern in reducing the edema volume in carrageenan-induced paw edema. At $500 \mathrm{mg} / \mathrm{kg} \mathrm{BW}$, CTA significantly suppressed the inflammation in paw edema compared to that of positive control, indicating by the low percentage of paw edema volume at 1,3 , and $5 \mathrm{hrs}$ after carrageenan injection. At gene level, qPCR data showed that CTA significantly down-regulated the expression of genes involved in PI3Ks, such as Akt1, PKB, Ilk, Pdk2, Pik3Ca, and $p 53$ in paw tissues. Thus, CTA may have a protective effect against edema via suppressing genes of PI3Ks.
\end{abstract}

\section{Introduction}

Inflammation is a body defense reaction to eliminate or limit the spread of injurious agent. Acute inflammatory response is characterized by an increase in vascular permeability and cellular infiltration leading to edema formation, as a result of extravasation of fluid and proteins and accumulation of leukocytes at the inflammatory site. Although it is a defense mechanism, the complex events and mediators involved in the inflammatory reaction can induce, maintain or aggravate several diseases (Amdekar et al., 2012). The phosphoinositide 3-kinase (PI3K) is a family of lipid kinases that have been shown to regulate several key events in the inflammatory response to damage and infection (Hawkins and Stephens, 2015). PI3K enzymes phosphorylate $\mathrm{PIP}_{2}$ to form $\mathrm{PIP}_{3}$ which stimulates the catalytic activity of Akt1, resulting in the phosphorylation of a host of other proteins that affect cell cycle entry, proliferation, survival, movement, oxidative burst, and cytokine gene activation. Inhibitors of PI3K disrupt the accumulation of $\mathrm{PIP}_{3}$ which in turn down-regulate the pro-inflammatory cytokines, thereby limit the inflammation, increase macrophage phagocytic ability, and resistance to infection (Koyasu, 2003).

Clitoria ternatea or butterfly pea flower is originated from Ternate island in the Moluccas (Indonesia) and 
belongs to a herbaceous perennial legume in Fabaceae family. C. ternatea is valued for its forage and medicinal importance. Its flower has a bright blue color due to the high accumulation of anthocyanins and anthocyanidins. Nair et al. (2015) reported that $C$. ternatea flower had ternatin anthocyanins (A1-A3, B1-B4, C1-C4, and D1D3) and phenolic flavonols (quercetin and kaempferol derivatives). Also, $C$. ternatea flower has been traditionally used for its pharmacological effects, such as anti-inflammatory, analgesic and anti-pyretic properties. In this study, $C$. ternatea anthocyanin extract (CTA) was evaluated for anti-inflammatory activities to reduce paw edema volume by suppressing gene expression related to PI3K signaling in carrageenan-induced rat paw edema.

\section{Materials and methods}

\subsection{Extraction of anthocyanin from dried Clitoria ternatea flower}

The flower petal of C. ternatea was collected from the cultivated field in Bogor, West Java province (Indonesia). Anthocyanin was extracted in methanol and acetone using maceration and lyophilization according to the modified method of Nair et al. (2015). A $100 \mathrm{~g}$ of dried flower petals was dissolved in $800 \mathrm{~mL}$ of methanol and acetone mixture (5:4). The solution was stirred for $24 \mathrm{hrs}$ at room temperature and solid particle was filtered using the filter paper. The solution was concentrated in a rotary evaporator at $180 \mathrm{rpm}$ and $45^{\circ} \mathrm{C}$, followed by lyophilization to obtain CTA.

\subsection{Sample preparation}

CTA at concentrations of 100,250 , and $500 \mathrm{mg} / \mathrm{kg}$ BW were dissolved in phosphate buffer saline (PBS) for oral feeding to rats. Ibuprofen standard (IBU) at $15 \mathrm{mg}$ / $\mathrm{kg} \mathrm{BW}$ was also dissolved in PBS. Sample was given orally to rats for 7 days.

\subsection{Intervention study}

Edema study was done using male Sprague-Dawley rats (Rattus norvegicus) weighing 75-100 gram that was housed in groups in a room. The photoperiods were daily adjusted to $12 \mathrm{hrs}$ of light and $12 \mathrm{hrs}$ of darkness. The environmental temperature was constantly maintained at $23-25^{\circ} \mathrm{C}$ with the humidity of $50-60 \%$. The animals were given access to standard laboratory rodent Chow and water ad libitum. The animals were acclimated to the animal facility for a week before being used in the experiment. The in vivo study was conducted at VStem Animal Facility (Bogor, Indonesia) and the protocol was approved by the Animal Welfare Supervision Commission and Use of Research Animals.

Edema in the right hind paw of the rat was induced by carrageenan injection (Ma et al., 2013). The rats were divided into six groups $(n=5)$ and orally administered with phosphate buffer saline (PBS, positive control and negative control), CTA (100, 250, and $500 \mathrm{mg} / \mathrm{kg} \mathrm{BW})$, and ibuprofen $(15 \mathrm{mg} / \mathrm{kg}$ BW) once every day for 7 consecutive days. One hour after the last intragastric administration, $50 \mathrm{~mL}$ of carrageenan $(1 \% \mathrm{w} / \mathrm{v}$ in PBS) was injected to the plantar side of the right hind paw, except the negative control group. the paw edema volumes were measured with a plethysmometer before the carrageenan injection and then $-1,1,3$, and $5 \mathrm{hrs}$ after injection. The inhibition of edema was assessed with the increase of paw volumes, which were calculated using the following formula: Increase of paw volume (\% I) $=($ Final volume - initial volume $) /$ initial volume $x$ $100 \%$. Five hrs after carrageenan injection, the animals were sacrificed. Tissue paw organs were collected and weighed, snap frozen in liquid nitrogen and stored at $80^{\circ} \mathrm{C}$ to be processed for preparation of homogenates.

\subsection{RNA isolation and $q P C R$ analysis}

Determination of genes related to PI3K signaling, including Akt1, Pik3Ca, Ilk, Pdk2, p53, and PKB was done using quantitative polymerase chain reaction (qPCR). Total RNA was isolated from rat paw organs using Trizol reagent (Life Technologies, California, USA) according to the manufacture's instruction and the primers were listed in Table 1. All primers were synthesized by Integrated DNA Technologies (Singapore). $\beta$-actin mRNA was used as the housekeeping gene. Values were then compared with those obtained by using the control-RNA obtained from each animal groups. The results were normalized to the housekeeping gene and fold change expression was calculated by using the threshold cycle $(\mathrm{Ct})$ values.

Table 1. Oligonucleotide primer sequences used in qPCR

\begin{tabular}{cllc}
\hline Primer & \multicolumn{1}{c}{ Forward } & \multicolumn{1}{c}{ Reverse } & Size $(\mathrm{bp})$ \\
\hline$A k t 1$ & GGACGGGCACATCAAGATAA & CTTCTCATGGTCCTGGTTGTAG & 217 \\
$P k b$ & CTCACAGAAGGGCCACATTT & AGCATCTGGACTGCTCTGGT & 228 \\
$I l k$ & GGAGAAGATGCAGGACAAGTAG & CCCATGTTGGGAGACATCAA & 240 \\
$P d k 2$ & CACTCTCCCTCCCATCAAAA & CCCTGGAAGTACTTGGCGTA & 222 \\
$P i k 3 c a$ & GCCAGATTTCATGGATGCTT & CCGTAAGTCATCGCCATTTT & 202 \\
$p 53$ & ACTGACAGCCTCTGCATCCT & GCAGAGACCCAGCAACTACC & 213 \\
$\beta$-actin & GTGCTATGTTGCCCTAGACTTC & TAGGAGCCAGGGCAGTAAT & 320 \\
\hline
\end{tabular}




\subsection{Statistical analysis}

Triplicate experiments were performed in this study. Data were expressed as the mean standard deviation (SD). The difference between treatment and the control group were analyzed using one-way analysis of variance (ANOVA). The probability of $p<0.05$ was considered statistically significant using $t$-test.

\section{Results and discussion}

\subsection{Effect of CTA in carrageenan-induced paw edema}

Inflammation is part of host defence mechanism against infectious agents and injury and it is also involved in the pathophysiology of many diseases when left unresolved. Acute inflammation is a short-term process which is characterized by typical signs of inflammation, such as swelling, heat, redness, pain and loss of function due to the infiltration of the tissues by plasma and leukocytes (Vetriselvan et al., 2013). Several pro-inflammatory mediators were released during the inflammatory response, including COX-2, TNF- $\alpha$, $\mathrm{NF} \kappa \mathrm{B}, \mathrm{IL}-7$, and IL- 6 and the induction of these proinflammatory mediators are largely regulated by transcriptional activation (Rommel and Camps, 2007). $C$. ternatea flowers are a rich source of anthocyanins and other flavonoids with anti-inflammatory activities. Anthocyanins modulate cell signaling, gene regulation, and other biological processes, which explain the health benefits of anthocyanins. Some studies have demonstrated that anthocyanin possess antiinflammatory activities by inhibiting the expression and biological activity of some pro-inflammatory substances like COX-2, iNOS, TNF- $\alpha$, NF- $\kappa$ B, IL-1b, IL-6, and many more (Miguel, 2011).

Our result demonstrated that the yield of CTA was $13.93 \% \mathrm{w} / \mathrm{v}$ compared to the weight of dried C. ternatea flowers. In order to determine the protective effect of CTA in acute-phase inflammation in vivo, a carrageenaninduced paw edema experiment was conducted. Carrageenan-induced rat paw edema model is a suitable test for evaluating anti-inflammatory drugs, which has frequently been used to assess the antiedematous effect of the drug. Carrageenan is a strong chemical use for the release of inflammatory and proinflammatory mediators (prostaglandins, leukotrienes, histamine, bradykinin, and TNF- $\alpha$ ). Carrageenan-induced edema is a biphasic response. The initial phase $(<1 \mathrm{hr})$ is due to the release of histamine, 5-hydroxytryptamine, leukotriens, kinins and cyclooxygenases in the first hour of the administration of carrageenan, and the delayed phase $(>1 \mathrm{hr})$ has been linked to the production of prostaglandins, bradykinin, neutrophil infiltration, and slow reacting substances which peaks at $3 \mathrm{hrs}$. The second phase is sensitive to both the clinically useful steroidal and nonsteroidal antiinflammatory agent (Vetriselvan et al., 2013).

Our results showed that there was a dose-dependent inhibitory activity in carrageenan-induced paw inflammation at all assessment times (Figure 1). Injection of carrageenan into the hind paw showed a time -dependent increase in paw volume, reaching its maximum at $3 \mathrm{hrs}$ after administration. CTA showed effect of down-regulating carrageenan-induced paw swelling compared with vehicle control group. CTA had a dose-dependent inhibitory activity in carrageenaninduced paw edema at all assessment times. At $500 \mathrm{mg} /$ $\mathrm{kg} \mathrm{BW}, \mathrm{CTA}$ treatment significantly reduced paw edema in rats (Figure 1).

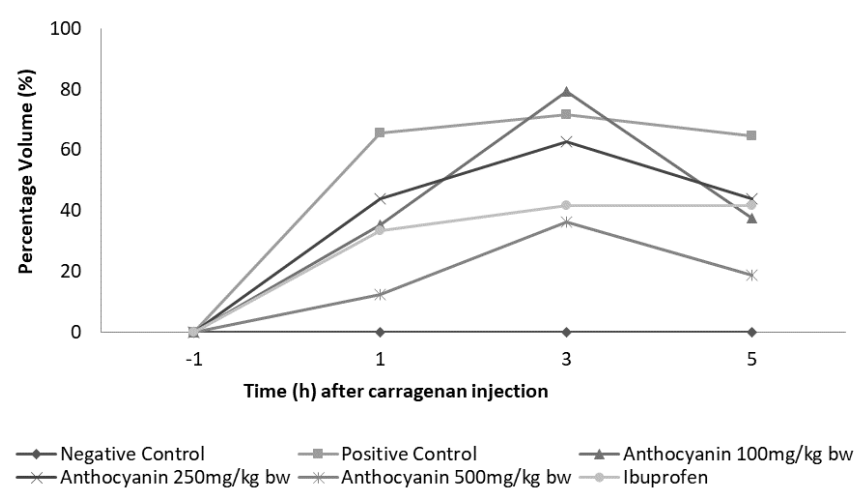

Figure 1. Effect of CTA administration on carrageenaninduced paw edema in rats. CTAs $(100,250$, and $500 \mathrm{mg} / \mathrm{kg}$ BW) and the vehicle were administered one hour prior to carrageenan injection. The rats were evaluated for paw edema at $\mathrm{t}=-1,1,3$, and $5 . \mathrm{n}=5$ for each group.

\subsection{Effect of CTA on gene expression related to PI3K signaling in carrageenan-induced paw edema}

Anti-inflammatory effects can be subdivided by site of action, the permeability of the vascular endothelium, or chemotactic effects on infiltrating immune cells. PI3K participate in signal transduction cascades that can directly activate and sensitize nociceptors and enhance pain transmission. These receptor families participate in signal transduction cascades, culminating in upregulation and/or activation of genes, and they also play essential roles in chemotaxis and immune cell infiltration leading to inflammation (Hawkins and Stephens, 2015). In this study, we evaluated whether CTA exerts its antiinflammatory effect in association with PI3K signaling pathway by evaluating genes that are involved in the pathway, such as $A k t 1, P K B, p 53, I l k, P d k 2$, and Pik3Ca in carrageenan-induced paw edema in rats. We found out that CTA significantly reduced the expression of $A k t l$, $P K B, p 53, I l k, P d k 2$, and $P i k 3 C a$ genes in carrageenaninduced paw edema in rats (Figure 2). Carrageenan had a stimulating effect against the expression of $A k t 1, P K B$, Ilk, Pdk2, Pik3Ca, and $p 53$ genes, and CTA treatment 
was found to significantly inhibit the expression of all genes involved in PI3K signaling in a dose-independent manner $(p<0.05)$. These results indicated that CTA exhibited potential protective effect against edema, and the efficacy of CTA at lowest dose was similar to standard (IBU).

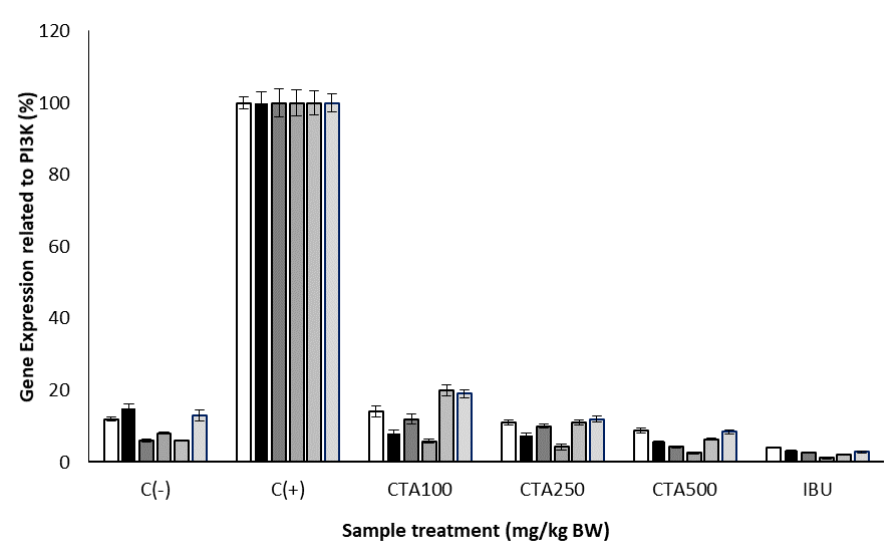

Figure 2. Effect of CTA administration on the gene expression of Akt1, PKB, p53, Ilk, Pdk2, and Pik3Ca in carrageenan-induced paw edema in rats. $\square$ negative control (C-); positive control (C+); $\square$ C. ternatea anthocyanin extract of $100 \mathrm{mg} / \mathrm{kg} \mathrm{BW}$ (CTA100); $250 \mathrm{mg} /$ kg BW (CTA250); $\square 500$ mg/kg (CTA500); and $\square$ ibuprofen standard $15 \mathrm{mg} / \mathrm{kg} \mathrm{BW}$ (IBU). $\mathrm{n}=5$ for each group.

The previous study demonstrated that both anthocyanins-rich extracts and anthocyanins effectively modulated gene expression of pro-inflammatory mediators in vitro and in vivo by their inhibition of NF$\mathrm{kB}$ and pro-inflammatory chemokine expression in monocytes (Mauray et al., 2010). Anthocyanins from Scutellaria radix also inhibited pro-inflammatory cytokines (IL-1 $\beta$, IL- 6 , TNF- $\alpha$, and NO) as well as COX2 (Chi et al., 2003). Treatment with PI3K inhibitors down-regulated the phosphorylation of Akt1 which was followed by attenuated expression of PKB, p53, Ilk, $\mathrm{Pdk} 2$, and $\mathrm{Pik} 3 \mathrm{Ca}$ in activated macrophages. The phosphorylation of Akt1 is often considered as a marker for PI3K activity. Full activation of Akt1 is dependent on phosphorylation at two sites, T308 and S473. Upon binding to PIP3, Akt1 is docked to the cell membrane which allows PDK2 and Ilk to also bound to the cell membrane to phosphorylate S473 in the 'activation loop' leading to partial Akt1 activation (Hemmings and Restuccia, 2012). Akt1 is critical for promoting microvascular leakage in response to inflammatory stimuli such as carrageenan and thus exerts a controlling influence over the magnitude of this response. The reduction in permeability reduces the influx of neutrophils and monocytes at the site of injury. Thus, the specific inhibition of Akt1 could provide a therapeutic approach to reduce vascular permeability and excessive leukocyte migration during carrageenan-induced acute inflammatory reaction or perhaps during conditions of excessive vascular leakage (Lorenzo et al., 2009). PI3K consists of heterodimers of a regulatory subunit, commonly the p85 and p110 catalytic subunit. Pik3Ca is responsible for coding the $\alpha$-catalytic subunit of class I PI3K that contains the binding site for the regulatory subunit, through which signals can be integrated from membrane receptors and activate the catalytic subunits. Activation and amplification of Pik3Ca gene have been associated with enhancing the Pik3Ca kinase activity and Akt1 phosphorylation (Durczyńska et al., 2012).

p53 has a crucial role in inflammation by controlling the expression of genes involved in apoptosis, cell cycle arrest, metabolism, and generation of pro-inflammatory cytokines. Macrophage polarization activates Akt1, which phosphorylates MDM2 gene and increases p53 ubiquitination. Increased tolerance to inflammation was associated with reduced p53 activity through inhibition of NF- $\kappa$ B and STAT1 signaling in macrophages, which further reduces the generation of pro-inflammatory genes and diminishes the macrophage phenotype ( $\mathrm{Li}$ et al., 2015). PKB is an important protease located downstream of PI3K. When activated by inflammatory factors, PI3K converts PIP2 into a second messenger PIP3 which can combine with the PH domain of PKB, phosphorylate it, and further cause changes of the target genes in the nucleus, and thereby regulate neutrophils activation, migration and the expression of inflammatory factors. This activation can mediate a large number of neutrophils infiltrating the inflamed tissue, release inflammatory mediators, and aggravate the inflammation.

Anthocyanin blocked intracellular signal transduction by inhibiting the activity of PI3K pathway, relieved the inflammation while protecting the tissue (Wei et al., 2015). The findings of our study showed that CTA administration inhibits the development of paw edema in rats. The gene expression of inflammatory mediators including Akt1, PKB, p53, Ilk, Pdk2, and Pik3Ca was increased in inflamed paw tissue and inhibited significantly in CTA received groups (Figure 2). Other study demonstrated that treatment with PI3K inhibitors (LY294002 and IC87114) had an ability to prevent acute inflammation in vivo by inhibition of Akt1 activation. Previous data also showed that several inflammatory mediators (i.e. IL-6, MCP-1, TNF $\alpha$ and iNOS/NO) of innate immunity are significantly upregulated by carrageenan. PI3K inhibitors downregulated the expression of cytokines and chemokines involved in innate immunity and in carrageenan-induced inflammation. These findings suggest that PI3K inhibitors could be used to attenuate inflammatory responses and that the mechanism of action behind this effect is the down-regulation of inflammatory gene 
expression (Eräsalo et al., 2015).

\section{Conclusion}

CTA exerts a protective effect and offers a novel therapeutic approach for the treatment of inflammatory diseases. CTA effectively reduced the volume of paw edema in rats by inhibiting the gene expression related to PI3K signaling pathway.

\section{Conflict of interest}

The authors declared there is no conflict interest. The authors alone are responsible for the content of the paper.

\section{Acknowledgement}

This research was funded by Decentralization Grant from General Directorate of Higher Education, Ministry of Research, Technology and Higher Education, Republic of Indonesia.

\section{References}

Amdekar, S., Roy, P., Singh, V., Kumar, A., Singh, R. and Sharma, P. (2012). Anti-inflammatory activity of Lactobacillus on carrageenan-induced paw edema in male Wistar rats. International Journal of Inflammation, 2012, $752015 . \quad$ https:// doi.org/10.1155/2012/752015.

Chi, Y.S., Lim, H., Park, H. and Kim, H.P. (2003). Effects of wogonin, a plant flavone from Scutellaria radix, on skin inflammation: in vivo regulation of inflammation-associated gene expression. Biochemical Pharmacology, 66(7), 1271-1278. https://doi.org/10.1016/S0006-2952(03)00463-5.

Durczyńska, K.W., Durczyńsk, A., Sporny, S., Strzelczyk, J. and Lewiński, A. (2012). Riedel's thyroiditis - A case report with genes' expression studies. Thyroid Research, 5, 2. https:// doi.org/10.1186/1756-6614-5-2.

Eräsalo, H., Laavola, M., Hämäläinen, M., Leppänen, T., Nieminen, R. and Moilanen, E. (2015). PI3K inhibitors LY294002 and IC87114 reduce inflammation in carrageenan-induced paw oedema and down-regulate inflammatory gene expression in activated macrophages. Basic and Clinical Pharmacology and Toxicology, 116(1), 53-61. https://doi.org/10.1111/bcpt.12284.

Hawkins, P.T. and Stephens, L.R. (2015). PI3K signaling in inflammation. Biochimica et Biophysica Acta, 1851(6), 882-897. https://doi.org/10.1016/ j.bbalip.2014.12.006.

Hemmings, B.A. and Restuccia, D.F. (2012). PI3K-PKB/ Akt pathway. Cold Spring Harbor Perspectives in
Biology, 4, a011189. https://doi.org/10.1101/ cshperspect.a011189.

Koyasu, S. (2003). The role of PI3K in immune cells. Nature Immunology, 4(4), 313-319. https:// doi.org/10.1038/ni0403-313

Li, L., Ng, D.S., Mah, W.C., Almeida, F.F., Rahmat, S.A., Rao, V.K., Leow, S.C., Laudisi, F., Peh, M.T., Goh, A.M., Lim, J.S., Wright, G.D., Mortellaro, A., Taneja, R., Ginhoux, F., Lee, C.G., Moore, P.K. and Lane, D.P. (2015). A unique role for $\mathrm{p} 53$ in the regulation of M2 macrophage polarization. Cell Death and Differentiation, 22(7), 1081-1093. https:// doi.org/10.1038/cdd.2014.212.

Lorenzo, A.D., Fernández-Hernando, C., Cirino, G. and Sessa, W.C. (2009). Akt1 is critical for acute inflammation and histamine-mediated vascular leakage. Proceedings of the National Academy of Sciences of the United States of America, 106(34), 14552-14557. https://doi.org/10.1073/ pnas.0904073106.

Ma, Y., Li, Y., Li, X. and Wu, Y. (2013). Antiinflammatory effects of 4-methylcyclopentadecanone on edema models in mice. International Journal of Molecular Sciences, 14(12), 2398023992.

Mauray, A., Felgines, C., Morand, C., Mazur, A., Scalbert, A. and Milenkovic, D. (2010). Nutrigenomic analysis of the protective effects of bilberry anthocyanin-rich extract in apo E-deficient mice. Genes and Nutrition, 5(4), 343-353. https:// doi.org/10.1007/s12263-010-0171-0.

Miguel, M.G. (2011). Anthocyanins: antioxidant and/or anti-inflammatory activities. Journal of Applied Pharmaceutical Science, 1(6), 7-15.

Nair, V., Bang, W.Y., Schreckinger, E., Andarwulan, N. and Zevallos, L.C. (2015). Protective role of ternatin anthocyanins and quercetin glycosides from butterfly pea (Clitoria ternatea leguminosae) blue flower petals against lipopolysaccharide (LPS)-induced inflammation in macrophage cells. Journal of Agricultural and Food Chemistry, 63, 6355-6365. https://doi.org/10.1021/acs.jafc.5b00928

Rommel, C., Camps, M. and Ji, H. (2007). PI3K delta and PI3K gamma: partners in crime in inflammation in rheumatoid arthritis and beyond? Nature Review Immunology, 7(3), 191-201. https://doi.org/10.1038/ nri2036

Vetriselvan, S., Subasini, U., Velmurugan, C., Muthuramu, T., Jothi, S. and Revathy. (2013). Antiinflammatory activity of Cucumis sativus seed in carrageenan and xylene induced edema model using albino wistar rats. International Journal of 
Biopharmaceutics, 4(1), 34-37.

Wei, M., Gong, Y., Tu, L., Li, J., Liang, Y. and Zhang,

Y. (2015). Expression of phosphatidylinositol-3

kinase and effects of inhibitor Wortmannin on expression of tumor necrosis factor- $\alpha$ in severe acute pancreatitis associated with acute lung injury. World Journal of Emergency Medicine, 6(4), 299-304. https://doi.org/10.5847/ wjem.j.19208642.2015.04.009. 